Musique et Sciences Sociales

Hors-série 1 | 2018

Musique, histoire, sociétés

\title{
Les universaux en musique et en musicologie
}

Réédition présentée par Márta Grabócz

\section{François-Bernard Mâche}

\section{OpenEdition}

\section{Journals}

Édition électronique

URL : http://journals.openedition.org/transposition/1717

DOI : 10.4000/transposition. 1717

ISSN : 2110-6134

Éditeur

CRAL - Centre de recherche sur les arts et le langage

Référence électronique

François-Bernard Mâche, "Les universaux en musique et en musicologie », Transposition [En ligne], Hors-série 1 | 2018, mis en ligne le 30 janvier 2018, consulté le 10 février 2020. URL : http:// journals.openedition.org/transposition/1717 ; DOI : 10.4000/transposition.1717

Ce document a été généré automatiquement le 10 février 2020.

\section{(c) (†) (2)}

La revue Transposition est mise à disposition selon les termes de la Licence Creative Commons Attribution - Partage dans les Mêmes Conditions 4.0 International. 


\title{
Les universaux en musique et en musicologie
}

\author{
Réédition présentée par Márta Grabócz
}

François-Bernard Mâche

\section{NOTE DE L'ÉDITEUR}

Article initialement publié dans ESCAL, Françoise et IMBERTY, Michel (eds), La musique au regard des sciences humaines et sociales, vol. I, Paris, L'Harmattan, 1997, p. 177-202.

François-Bernard Mâche (né en 1935) est l'un des compositeurs français les plus originaux et innovants de sa génération. Ses idées et ses œuvres ont marqué considérablement l'évolution de la musique contemporaine des soixante dernières années.

Sa position est très exceptionnelle dans la mesure où il est à la fois compositeur, philosophe de la musique et fin connaisseur de l'Antiquité. Il est donc un compositeur qui est aussi un musicologue, un zoo-musicologue qui est aussi un esthéticien ; il est un linguiste qui s'intéresse aussi au fonctionnement du système nerveux, du cerveau, et à la psychologie cognitive ; un mythologue qui analyse aussi des sons enregistrés à l'aide des sonagrammes ou des logiciels. De ses activités multiples résulte un œuvre qui regroupe environ cent quinze opus musicaux, six ouvrages, quelque cent quatre-vingts articles et quatre directions de recueil sur la création contemporaine.

Dès 1970, il est le père du courant appelé « naturalisme sonore » qui a plusieurs représentants, y compris dans la jeune génération des compositeurs de différents pays; il est l'inventeur des notions de " zoomusicologie » et de " phonographie " dans les années 1970 ; il est le premier à avoir réalisé une œuvre « spectrale » en transcrivant à l'orchestre les sonorités - d'après une analyse par sonagramme - 
d'un poème récité de Paul Eluard (Le son d'une voix, 1964); il a créé une méthodologie pour l'examen des archétypes sonores, etc. Ses livres traduits en plusieurs langues ont contribué au développement de la musicologie, de la sémiotique et de l'esthétique musicales au niveau international.

Dans son parcours, l'activité de théoricien va toujours de pair avec celle du compositeur, du créateur. En 1957, il obtient son diplôme d'Études supérieures d'Archéologie grecque, et son Duo pour violon et piano est primé et exécuté au festival de Bilthoven en Hollande. En 1958, il est agrégé de Lettres classiques (ENS) et il entre au GRM, mais il est également admis dans la classe de Messiaen au CNSM de Paris. Ses premières œuvres vocales, instrumentales et électroacoustiques sont créées dès 1959. Il est directeur du GRM en 1962. Dès les années 1970-1980, ses créations sont honorées de plusieurs prix de compositions importants (par exemple : Prix ltalia 1977 ; Grand Prix international de la musique 1988; commandeur des Arts et des Lettres en 1990 ; Grand Prix de la musique symphonique de la SACEM 2002, etc.). En 1980, il obtient le diplôme du docteur d'État ès lettres et Science humaine avec une thèse (sous la direction de I. Xenakis) sur L'idée du modèle en musique aujourd'hui à l'université de Paris I. Il est compositeur invité et professeur de master-classes dans une vingtaine de pays. Entre 1983-1993, il est professeur de musicologie à l'Université de Strasbourg II, où il y crée le centre PRIMUS, formation exceptionnelle pour les ingénieurs du son. En 1983 il publie Musique, mythe, nature ou les dauphins d'Arion chez Klincksieck, dont la troisième édition est sortie chez Adeam Musicae en 2015 (livre également publié en anglais et en italien) ${ }^{1}$. En décembre 1993, il est élu directeur d'études à l'EHESS, avec un programme de recherche consacré aux « Universaux musicaux ».

Le 18 décembre 2002, il est élu à l'Institut de France, Académie des Beaux-Arts, au fauteuil de Iannis Xenakis. Le 26 octobre 2004, il présente un discours à la séance solennelle de rentrée des Cinq Académies. En 2011, il est nommé docteur honoris causa à l'Université d'Athènes, la célébration étant accompagnée d'une masterclass et d'un concert.

Concernant ses livres, en 2001 il publie Musique au singulier chez Odile Jacob où il introduit et met en contexte les notions d'universaux, d'archétypes, de génotypes et phénotypes ${ }^{2}$; en 2006 paraît un autre livre important accompagné d'un DVD : François-Bernard Mâche. La musique, la nature, les oiseaux. Entretien avec Bruno Serrou ${ }^{3}$. En 2012 son ouvrage intitulé Cent opus et leurs échos, offre l'intégralité des présentations ou des notices sur ses œuvres, rédigées par lui-même en deux temps, parfois avec un intervalle de 30-40 ans (il s'agit des pièces écrites jusqu'en 2011) ${ }^{4}$.

Ses séminaires à l'EHESS assurés jusqu'en 1999 étaient consacrés aux thèmes suivants : « Le modèle en musique », « Les universaux en musique », « La répétition en musique », etc.

Le 10 février 1994, il intervient au colloque de l'EHESS avec une communication intitulée "Les universaux en musique et en musicologie », article publié plus tard dans les actes du colloque dirigés par Françoise Escal et Michel Imberty.

Ce texte - reproduit ici - a un rôle central dans la production scientifique et même dans la création musicale de François-Bernard Mâche. Même si sa pensée a 
considérablement évolué depuis 1994 (voir les livres, les recueils mentionnés plus haut), cet article souligne l'état de la réflexion musicologique dans les années 1990, et place sa propre recherche et création dans ce contexte, tout en esquissant des perspectives nouvelles.

La première partie, offre un tour d'horizon critique de la situation de la musicologie parmi les sciences humaines. Selon le compositeur, la musicologie essaie en vain d'acquérir le rang d'une discipline autonome, car selon les expériences du passé, elle s'appuie sur telle ou telle discipline (l'histoire, la sociologie, la sémiologie, les sciences cognitives, la psychologie, les

mathématiques, etc.), tout en restant dépendante de ses consœurs. Mâche suggère à la musicologie de ne pas vouloir progresser uniquement selon la dépendance des autres disciplines qui évoluent, mais de faire un effort en vue d'une synthèse originale pour acquérir l'autonomie.

L'autre problème grave que doit combattre la musicologie, est son retard par rapport à l'évolution de la musique, de la connaissance du répertoire musical des compositeurs, des anthropologues, du public. La musicologie était victime de « la conviction ancienne que seule la musique européenne et ses dérivés étaient conformes à l'acoustique naturelle » (ou à la résonance naturelle) laquelle est devenue le seul cadre conceptuel, offrant un langage omnipuissant, permettant de penser la musique. Après 1948, avec l'usage du magnétophone et l'évolution des techniques d'enregistrement, l'écho des grandes traditions musicales d'Afrique et d'Asie a atteint l'Europe qui a poussé les anthropologues et les ethnomusicologues à découvrir la relativité des valeurs esthétiques et celle du vocabulaire musicologique. «La découverte de la relativité des cultures autorisait la relativité des esthétiques, et ce vertigineux "tout est donc permis" a émergé très vite à la conscience des compositeurs " (page 6 sur 26). Les valeurs que l'Europe avait crues universelles ne l'étaient donc pas, du moins sous la forme qu'on leur prêtait, et la musicologie devait admettre la diversité des langages, leur communication et leur «traduction».

Entre les années 1950-1970, une modernité radicale, une idéologie essentiellement culturaliste a jeté la suspicion sur l'idée que des valeurs puissent être universellement partagées, et particulièrement sur la problématique très ancienne des universaux. Mais à partir d'environ 1975, les travaux de George List, Klaus Wachsmann, Dane L. Harwood, Gilbert Rouget ont fait ressurgir la problématique des universaux. À l'instar des méthodes de Claude Lévi-Strauss, Zellig S. Harris et Nicolas Ruwet, Mâche a commencé d'explorer l'analyse distributionnelle.

Une deuxième partie de l'article explique les démarches du compositeur et du théoricien à partir des années 1960. Dès 1963, il a déposé un projet (au GRM) qui tentait d'appliquer les méthodes de type structuraliste à l'analyse des musiques électroacoustiques et autres. Dans ses œuvres des années 1960 également, la partition illustrait l'application d'une approche analytique : une des nouveautés étant qu'un contexte esthétique spécifique pouvait permettre de transformer les signaux sonores en signes musicaux... À ce moment-là, le compositeur et le musicologue focalisait sa recherche sur les universaux dans le but de pouvoir légitimer les choix compositionnels. Son article sur les « Méthodes linguistiques en 
musicologie » a donné le ton en $1971^{5}$. Puis les chapitres de son livre Musique, mythe, nature de 1983 portant sur la zoomusicologie et les modèles ont montré d'autres méthodes explorant des traits structurels, des formes sonores, des processus sonores ${ }^{6}$. Il a progressivement découvert que les concepts de Roger Caillois ou de la psychanalyse (Jung, Diel) pouvaient aider à interpréter les troublantes convergences que le musicien constate entre les structures sonores animales et les musiques humaines. Il a été alors amené « à adopter à titre d'hypothèse générale l'idée qu'une part des formes musicales, et notamment celle qui paraît commune aux différentes cultures, et même aux animaux, pouvait reposer sur l'existence de [...] schèmes spontanés issus du système nerveux central».

Dans la troisième partie de l'article, il propose un nouveau type de recherche, à l'explorer également au sein de l'EHESS, basée sur la quête des phénotypes, génotypes et archétypes. Cette recherche répondrait aussi au manque d'autonomie de la musicologie et à son ambition d'intégration ou de synthèse à partir des différents moyens d'action dont elle dispose sur le plan intellectuel. La toute dernière partie de l'article expose les grandes catégories de sa recherche : 1/les catégories formelles (avec cinq sous-groupes, comme par exemple les échelles musicales ; les modèles narratifs comme la crise, l'énumération, le conflit, la variation évolutive ; les modèles physiques tels que le reflux, l'écho, le ruissellement, l'éclatement, l'appel etc.) et 2/les données de l'imaginaire musical avec cinq sous-catégories comme par exemple les symboliques sexuelles (masculinféminin dans les rythmes, les contours, les accents ; tabous organologiques etc.); les symboliques cosmologiques (heures, saisons, points cardinaux, astrologie etc.) ; les symboliques émotionnelles (ethos, couleurs, circonstances etc.). La coda de l'article souligne l'importance d'une musicologie comparée qui pourrait faire face aux défis de l'évolution des sciences humaines.

Márta Grabócz (université de Strasbourg)

1 Après avoir passé des décennies au service de l'Histoire, la musicologie cultive une ambition permanente : acquérir parmi les Sciences humaines le rang d'une discipline autonome. C'est peu de dire qu'elle est encore assez loin d'avoir atteint cet objectif supposé. On l'a vue selon les époques beaucoup plus souvent miser presque impulsivement sur telle ou telle voie de recherche que proposer après mûre réflexion aux autres sciences une méthodologie originale. Tantôt l'histoire ou la sociologie, tantôt la sémiologie, et maintenant le développement des sciences cognitives, sont venus alimenter ses recherches et ses espérances, sans que les services rendus par ces consœurs aient pour l'instant été payés de retour. Tant qu'il en sera ainsi, c'est-à-dire tant que la musicologie dépendra, dans ses méthodes comme dans ses objectifs, d'autres disciplines, il semble raisonnable de penser qu'elle devra chercher à progresser non seulement en se lançant sur les voies ouvertes par d'autres sciences humaines, mais aussi, comme premier pas vers l'autonomie, en intégrant autant que possible leurs acquisitions dans un effort de synthèse original. 
2 Il n'est donc pas question, dans l'état de dépendance actuel de la musicologie, de pouvoir se passer de tout ce que lui enseignent l'histoire, doyenne d'âge des Sciences humaines, non plus que la sociologie, l'analyse structurale, la psychologie expérimentale et les Sciences cognitives en général, ou encore les multiples branches des mathématiques. Mais on pourrait peut-être, tout en vivant ainsi sur le crédit ouvert généreusement par les autres sciences, et, oserai-je dire, en improvisant avec plus ou moins de bonheur sur leur basse obstinée, s'accorder sur l'objectif assez modeste d'une harmonisation de leurs apports.

3 Si nous en sommes là, c'est que la musique a avancé d'un pas beaucoup plus vif que la musicologie. Lorsque le langage tonal avait atteint assez de stabilité pour faire l'objet d'une grammaire systématique, la musicologie pouvait s'appuyer sur des règles (avec leurs exceptions et leurs transgressions dûment repérées), et légiférer en toute bonne conscience. Dès le début $\mathrm{du} \mathrm{xx}^{\mathrm{e}}$ siècle, on a dû constater que des compositeurs refusaient de jouer vis-à-vis des règles le jeu traditionnel de l'oscillation entre soumission et révolte, et prétendaient soit se passer de règles, comme Debussy, soit en inventer un nouveau corpus complet et en état de marche, comme Schönberg.

4 La musicologie a tenté de penser selon ses méthodes les plus éprouvées l'afflux troublant d'innovations radicales. Elle a, parfois jusqu'à nos jours, tenté, à l'aide de son article de foi fondamental, la résonance naturelle, d'interpréter ces innovations comme généralisations progressives d'une même démarche historique, consistant à intégrer les dissonances dans une conscience harmonique prise comme norme. À l'arrière-plan de cet effort de récupération, la conviction ancienne que seule la musique européenne et ses dérivés était conforme à l'acoustique naturelle, les musiques atonales ou exotiques ne pouvant être que le fruit d'une coupable ignorance de ses lois. D'où, au début du $\mathrm{Xx}^{\mathrm{e}}$ siècle, les hypothétiques accords de $11^{\mathrm{e}}$ et de $13^{\mathrm{e}}$ ajoutés à l'arsenal des dissonances reconnues, et dans l'entre-deux-guerres les aventureuses théories sur les harmoniques inférieurs.

5 Ce qui accusait davantage encore le retard, devenu habituel, de la théorie musicologique sur l'actualité de la musique, c'est qu'à la suite de l'expansion coloniale de l'Europe à travers le monde, les informations sur d'autres cultures venaient jeter l'inquiétude, et peu à peu le doute radical, concernant le bien-fondé du monopole musical que l'Europe s'était arrogé, et qu'elle croyait depuis deux siècles fondé dans sa dimension universelle sur la nature même du son. La musique a d'ailleurs mis plus de temps à douter de son ouie que les arts plastiques n'en ont mis à réformer le regard. Tant que l'enregistrement n'a pas acquis les facilités de mobilité et de durée que le magnétophone est venu lui donner après 1948, l'écho des grandes traditions musicales d'Asie et d'Afrique atteignait difficilement l'Europe, et peu de compositeurs comme Delage ou Bartók avaient eu un contact direct avec d'autres pensées musicales.

6 De plus cette découverte remettait souvent en cause non seulement les valeurs esthétiques européennes, mais jusqu'au cadre conceptuel qui lui permettait de penser la musique. Non seulement d'autres peuples jugeaient différemment ce qu'ils entendaient, mais encore leurs langages et leurs coutumes situaient autrement que nous ce que nous appelons musique, cuvre, ou concert.

7 Les anthropologues, au lendemain de la Deuxième Guerre mondiale, découvrant à la fois la relativité des valeurs esthétiques et celle du vocabulaire musicologique, ont bien souvent récusé les unes et les autres pour s'en tenir prudemment à des analyses et à des descriptions qui s'abstiendraient autant que possible de tout préjugé et même de tout 
jugement, avant de tenter comme ils le font aujourd'hui de donner la parole aux autres à propos de leurs propres pratiques. Si par exemple les cultures africaines, ou la musique balinaise, ont pu à la fois offrir des phénomènes sonores et sociaux hautement complexes sans apparemment présenter le plus souvent de recul théorique sur leurs pratiques, il est, selon plusieurs ethnologues, du devoir de l'enquêteur de détecter dans les discours de ces praticiens la théorie implicite sur laquelle ils s'appuient, et de la formuler clairement dans une des langues en usage dans le monde de la recherche scientifique.

8 Le goût pour l'exotisme du $\mathrm{XIX}^{\mathrm{e}}$ siècle et du début $\mathrm{du} \mathrm{xx}^{\mathrm{e}}$ siècle avait été la première réaction de l'Europe à la révélation d'un ailleurs musical. De Saint-Saëns à BourgaultDucoudray les compositeurs ont appris à ouvrir l'oreille. L'analyse et l'assimilation ont marqué la seconde étape au temps de Stravinski, Bartók, Jolivet et Messiaen. Mais pendant ce temps, la réflexion musicologique hors du cercle des compositeurs était relativement timide, comme la lecture de certains articles de l'Encyclopédie Lavignac le montre bien. Avec l'enregistrement, la musique concrète, et les voyages en avion, l'afflux d'informations a peu à peu brassé dans le magma d'une world music toutes les richesses du monde désormais proposées comme biens de consommation. Le métissage généralisé achève de brouiller la connaissance des spécificités locales. Là encore la musicologie accuse son inévitable retard: elle est maintenant très consciente de la relativité culturelle. Un demi-siècle trop tard, car celle-ci n'empêche plus des emprunts croisés tellement fréquents et généralisés que bientôt les preuves de cette relativité ne seront plus accessibles que dans des archives.

9 L'apparition de musiques atonales au début de ce siècle avait été maintes fois interprétée en fonction d'une évolution historique remontant à Wagner et à Liszt. Mais ce développement dans le champ mi-clos de l'Histoire européenne ne tenait pas compte de la conscience, plus précoce chez les artistes que chez les théoriciens, de la relativité des esthétiques. Rodin collectionnait des statues africaines et océaniennes avant que la réflexion de Malraux ne les intègre dans un Musée imaginaire. De même Debussy et Varèse écoutaient les musiques des Expositions universelles tout autrement que les pédagogues même les plus ouverts. La découverte de la relativité des cultures autorisait la relativité des esthétiques, et ce vertigineux « tout est donc permis » a émergé très vite à la conscience des compositeurs.

10 Le montage fait par Pierre Schaeffer sous le nom de Simultané camerounais en juin 1959 à la salle Gaveau, tout comme Orient-Occident composé l'année suivante par Xenakis pour le film de Fulchignoni à l'UNESCO, poursuivaient cette découverte avec les nouveaux moyens apportés par la bande magnétique. Si tout au long $d u x^{e}$ siècle un dialogue s'est développé entre les cultures, tandis que l'hybride particulièrement vivace du jazz s'imposait comme la plus populaire des musiques un peu partout, c'est bien que les valeurs que l'Europe avait crues universelles ne l'étaient pas, du moins sous la forme qu'on leur prêtait. Mais c'était aussi qu'entre ces langages, dont on avait fini par admettre la diversité, une communication, et des traductions, se révélaient possibles.

11 Or à cette époque, c'est-à-dire à partir des années 50 environ, une idéologie radicalement culturaliste, en musicologie comme ailleurs, tentait de jeter la suspicion sur l'idée que des valeurs puissent être universellement partagées, et particulièrement sur la problématique très ancienne des universaux. Échaudée, pour ainsi dire, par l'expérience d'une surdité coupable qu'elle prenait conscience d'avoir entretenu pendant des décennies à l'égard d'autres systèmes de valeurs musicales, la musicologie 
non seulement tenait pour désormais acquis que toute prétention à fonder en nature les bases de la musique était une illusion devenue caduque en même temps que la tonalité, mais encore poussait le relativisme culturel jusqu'à promouvoir la singularité esthétique comme gage d'authenticité : puisqu'il y avait autant de systèmes que d'aires culturelles, pourquoi n'y aurait-il pas autant de «langages" que d'œuvres individuelles? La musicologie se sentait alors confortée dans ce relativisme par l'émergence d'une génération de compositeurs théoriciens très prolixes, qui lui promettaient, pourvu qu'elle se mît à leur service, d'opérer un aggiornamento inespéré, grâce auquel elle parviendrait enfin à penser « en temps réel » la musique en train de se faire. Cette modernité radicale a tenu le haut du pavé au moins jusqu'au milieu des années 70.

L'interaction des interprétations anthropologiques et des credo modernistes a alors repoussé au second plan la question des universaux. Les ethnomusicologues, soucieux à juste titre de ne pas appliquer à leurs domaines d'études des catégories sans pertinence réelle en dehors de la civilisation occidentale, ont souligné tout ce qui dans les pratiques musicales traditionnelles relevait d'un autre champ sémantique que ce que l'Occident entend par musique, au point de décrire les aspects sonores de certaines conduites comme une simple composante d'un comportement global, sans spécificité légitime. Non sans quelque naïveté, certains artistes, apparemment séduits, ont promu dès lors une pratique de performance qu'ils jugeaient plus conforme à cette intégration traditionnelle de la musique dans un art total, et sont venus ainsi récuser activement dans le cadre de la civilisation occidentale les notions de musique et même d'art.

13 Cependant au sein même des questionnements favorisés par les travaux des ethnomusicologues est apparue une résurgence de la problématique des universaux. List et Wachsmann l'ont ranimée dès 1971. En 1976 Dane Harwood a reposé la question selon la perspective de la psychologie cognitive. Gilbert Rouget s'est intéressé à la répétition comme universel, à propos d'un chant béninois. Tandis que dans les années 60, des études comme celle de Marius Schneider et de Walter Wiora tendaient surtout à élargir les concepts issus de la résonance naturelle, les recherches menées depuis une trentaine d'années tentent de s'appuyer sur d'autres fondements: psychologiques avec les travaux d'Imberty, épistémologiques avec ceux de Nattiez, esthétiques avec Revault d'Allonnes. La musicologie comparée, dont l'ethnomusicologie s'était fortement démarquée en la dénonçant comme illusoire ou déplacée, est peutêtre sur le point de retrouver une certaine légitimité, qui ne prétendrait pas remettre en cause les acquis anthropologiques, mais qui réévaluerait plutôt leur portée. La relativité des cultures musicales n'est après tout pas telle qu'on y voie des systèmes fermés les uns aux autres. Une certaine part d'universalité s'y rencontre, tout comme dans les fondements du langage en général.

14 Depuis les apports exemplaires de Levi-Strauss et de Z. Harris, l'anthropologie et la linguistique structurales ont montré à la musicologie un chemin qu'elle s'est essayée à suivre de diverses manières. Chez Ruwet, Nattiez et moi-même, l'analyse distributionnelle a été une des premières voies explorées. Mais l'essoufflement rapide de ce type de méthodes inductives, leur mouvement du terrain à la loi s'arrêtant souvent à mi-course, a conduit à miser plutôt sur les modèles chomskyens, hypothético-déductifs, pour une recherche sur les universaux. L'absence de niveaux comparables à ceux de la linguistique et la définition introuvable des mélèmes ont alors conduit certains à se détourner de l'analyse des messages pour expérimenter des 
modèles génératifs. Mais là aussi il est souvent bien difficile de faire se rejoindre la théorie et les réalités musicales. Les difficultés de la traduction automatique et celles d'une définition des bases communes à tous les langages musicaux sont assez analogues.

Ce tour d'horizon prélude à des propositions d'orientation, qui se situent à certains égards dans le prolongement lointain de certaines préoccupations des années 1960. C'est seulement pour mieux expliquer ces propositions que je me permettrai quelques considérations autobiographiques, de celles qu'en général on pardonne plus aisément à un compositeur qu'à un homme de science. À cette époque, mon activité au Groupe de Recherches musicales (GRM) m'avait mis en présence d'une démarche analytique qui tentait, avec A. Moles et P. Schaeffer, de créer une typo-morphologie universelle pour l'analyse de toute structure sonore. Malgré son intérêt, ce corpus d'outils analytiques, rassemblé quelques années plus tard dans le Traité des objets musicaux, avait le grave défaut de ne prendre en compte ni le contexte ni le devenir de ces objets, par ailleurs plutôt sonores que musicaux. Ce qui était présenté sous le nom de « solfège » n'offrait guère que des vertus taxinomiques. Persuadé par l'étude de la phonologie qu'il s'agissait là d'une approche purement «étique » selon la terminologie de J-J. Nattiez, donc insuffisante, j'avais été conduit à déposer en janvier 1963 un projet qui, selon une voie différente, de type "émique", tentait d'appliquer à l'analyse des musiques électroacoustiques et autres des méthodes de type structuraliste. Le refus de ce projet me conduisit alors à démissionner de la direction que m'avait confiée P. Schaeffer.

Quelques années plus tard, l'article Langage et musique, paru en 1969, coïncidait avec une innovation radicale dans le domaine de mes compositions: avec l'œuvre Rituel d'oubli pour orchestre et bande magnétique j'intégrais pour la première fois des bruits bruts enregistrés (sons animaux, langues rares, bruits de guerre, sons des éléments) à une écriture instrumentale dérivée de ces modèles. Contrairement à la façon dont les compositeurs antérieurs, tels Messiaen, et moi-même jusqu'alors, avions procédé, le modèle sonore ne se situait plus seulement en amont de l'œuvre, et la représentation faisait place, grâce aux nouvelles possibilités techniques, à la présentation. D'une certaine manière, la partition illustrait l'application d'une approche analytique tendant à démontrer, presque comme un manifeste, que la prétendue incompatibilité des bruits "anecdotiques" et de la musique cessait d'exister si un contexte esthétique spécifique réussissait à transformer les signaux sonores en signes musicaux. Dès lors le souci de rechercher quels universaux pouvaient soit légitimer soit au moins expliquer cette alliance qui était au centre de mes projets de compositeur m'a conduit à publier diverses réflexions reliées de plus ou moins près à ce problème.

Mes études littéraires classiques m'ayant donné quelques bases de linguistique structurale, j'ai proposé, dans un article paru en 1971 dans la revue Musique en jeu, la première analyse de type structural d'une musique polyphonique, sur des partitions de Monteverdi et de Varèse. Sans être nécessairement rattachée à la problématique des universaux, cette méthode analytique apparaissait comme la plus générale qui soit, et propre à rendre compte aussi bien de musiques modales que d'œuvres qui, comme celles de Varèse, ressortissent à des catégories et à des organisations inédites. Ce sont d'ailleurs les ethnomusicologues qui se sont montrés les plus intéressés à son usage, pour son aptitude à dépasser les catégories classiques de la musique européenne.

L'utilisation de modèles parlés et de modèles animaux dans certaines de mes œuvres musicales à partir de 1969 (Korwar, Naluan, Uncas etc.) devait m'amener à employer la 
même méthode d'analyse pour les uns et les autres. La situation qui avait conduit les linguistes américains du cercle de Harris à développer l'analyse distributionnelle se reproduisait de manière encore plus radicale avec les chants animaux : de même que les linguistes devaient tenter une analyse phonologique de leurs corpus de langues indiennes enregistrées sans pouvoir faire appel à des tests sémantiques de commutation des phonèmes, de même j'étais par définition privé d'informateur devant les modèles animaux choisis pour leurs potentialités musicales. En procédant aux mêmes opérations de segmentation, de recherche paradigmatique, d'opposition des invariants et des processus de variation, sur des documents tantôt « naturels " tantôt linguistiques, j'ai été amené à constater des phénomènes inattendus de convergences beaucoup plus étroites qu'on ne l'imaginait. Après quelques années d'approfondissement de ces analyses, grâce à l'appui du Pr Busnel et de ses appareils du Centre de Recherches Zootechniques de Jouy-en-Josas, j'ai présenté les bases de ce que j'ai baptisé Zoomusicologie dans un chapitre de mon ouvrage Musique, Mythe, Nature. Seule une minorité de spécialistes d'acoustique animale (Hartshorne, Thorpe, J. HallCraggs, P. Szöke entre autres) prennent en compte l'hypothèse d'une dimension esthétique des signaux animaux. Les autres s'en tiennent en général à une stricte perspective éthologique, et s'intéressent dans la communication animale aux modes d'émission et de réception, en excluant plus ou moins de leurs préoccupations la diversité phonétique et syntactique des messages, considérée comme pure redondance. Or l'analyse de la syntaxe de ces signaux à l'aide des outils tels que sonagraphes, détecteurs de hauteurs, logiciels de notation etc., et conduite par un musicien, met en évidence une quantité de traits structurels (dans les formes sonores) et structuraux (dans les processus) trop importante pour qu'on y voie une convergence anodine et seulement pittoresque. L'importance de l'innovation individuelle chez certaines espèces chanteuses où l'ontogenèse l'emporte de beaucoup sur la phylogenèse conforte l'hypothèse que, loin d'être un phénomène culturel aussi spécifique que la parole, la musique, au sens minimal d'une organisation jouant sur et avec les sons, est peut-être partiellement une fonction biologique qui dépasse les limites de l'espèce humaine. La polémique soulevée dans Musique en jeu en 1971-1972 par mon article sur J. Cage tenait en partie à ma conviction grandissante que, si toute écoute était potentiellement musicale, il n'en existait pas moins des formes sonores prédéterminées pour un tel rôle ; position à la fois voisine et très différente de celle de Cage.

Il m'est alors apparu que peut-être les traditions mythologiques qui si fréquemment, tant dans le chamanisme que dans l'ornithomancie grecque ou les traditions germaniques dont Wagner s'est souvenu dans Siegfried présentent le monde sonore des animaux comme délivrant des messages cryptés, que seuls quelques devins savent entendre, voulaient peut-être nous dire symboliquement à leur manière cette proximité de l'imaginaire sonore humain et animal. J'ai dès lors été conduit à exploiter, tant dans mes recherches théoriques (La musique égale du mythe, par exemple) que dans mes œuvres, ma formation d'helléniste, et également à voir dans quelle mesure les concepts de R. Caillois ou de la psychanalyse (Jung, Diel) pouvaient aider à interpréter ces troublantes convergences que le musicien constate entre les structures sonores animales et les musiques humaines.

Non seulement les mythes nous disent «le temps où les animaux parlaient» mais ils nous le disent dans des langues qui n'ont pas été historiquement en contact, pour autant qu'on le sache. L'hypothétique diffusionnisme, qui de toute manière n'explique pas pourquoi toute autre trace de contact aurait disparu, apparait souvent moins 
plausible que l'hypothèse d'une naissance spontanée des mêmes schèmes narratifs, sonores ou gestuels en de multiples lieux culturellement indépendants. Qu'on accepte ou non de les appeler archétypes, il semble qu'ils existent au niveau d'une imagination mythique spontanée, préalablement à tout récit mytho-logique et à toute élaboration rituelle, sonore ou gestuelle. Des œuvres comme Danaé, Kassandra ou Andromède traduisent à leur manière ces réflexions, que je me propose de faire avancer également sur le plan théorique.

21 J'ai alors été amené à adopter à titre d'hypothèse générale l'idée qu'une part des formes musicales, et notamment celle qui paraît commune aux différentes cultures, et même aux animaux, pouvait reposer sur l'existence de tels schèmes spontanés issus du système nerveux central. Cette hypothèse s'est trouvée confortée par sa convergence avec les théories de J-P. Changeux qui a bien voulu y voir une approche différente de certains concepts qu'il appuie sur ses travaux de biologiste. Une autre convergence, découverte après coup, apparaît aussi en partie avec les études qu'a faites Gilbert Durand des Structures anthropologiques de l'imaginaire.

Il semble donc que par des approches très diverses plusieurs chercheurs redonnent aujourd'hui droit de cité à un cadre épistémologique qui puisse accueillir l'hypothèse d'universaux musicaux, en explorant des voies de recherche plus diversifiées que la seule résonance naturelle. Il me semble aussi que la reconnaissance de composantes naturelles au sein des faits de culture n'est plus frappée d'un anathème, voire d'une suspicion politique, aussi rigoureux qu'il y a quelques années. Les oppositions qu'ont pu soulever en leur temps certains de mes écrits comme Le réalisme en musique (1960), Une ville sonore (1971), Xenakis et la nature (1972), Considérations biomusicologiques (1982), ou La musique au naturel (1987) semblent se faire moins nombreuses ou moins violentes. Le type de recherche que je me propose de pratiquer très prochainement dans cette École me semble répondre, même si ce n'est qu'une réponse parmi d'autres possibles, à la fois au manque d'autonomie de la musicologie, et à sa légitime ambition d'accéder à une intégration, ou même à une synthèse, des différents moyens d'action dont elle dispose sur le plan intellectuel.

23 Trois catégories d'universaux, qui sont sans doute aussi trois niveaux, sont à mettre en évidence :

24 - les phénotypes sonores et musicaux. Notre oreille nous présente des sons et des figures qui manifestent des ressemblances. Percevoir ces ressemblances est à la portée de tout musicien exercé. En évaluer la portée relève d'une réflexion méthodique dont on proposera ci-dessous quelques modalités. Il est certain que les risques de malentendu sont nombreux entre contextes historiques et/ou culturels différents pour un même phénotype supposé. Les perspectives ouvertes par la psychologie cognitive en musicologie peuvent-elles confirmer, par une tout autre approche, celles que le structuralisme a tenté de dégager depuis un quart de siècle, et que les méthodes historiques avaient plus anciennement ouvertes? L'enjeu de cette synthèse qui, on l'aura reconnu, se joue entre l'esthésique, le niveau neutre et le niveau poiétique chers à Molino, passe par un certain éclectisme des approches. Sans perdre de vue (d'oreille...) la figure sonore à identifier, il importe de la cerner tantôt avec les moyens de la psychologie cognitive, pourvu qu'elle réussisse à nous apprendre comment se constitue une figure sonore dans notre conscience, tantôt avec ceux de la description plate de l'analyse distributionnelle, et tantôt enfin avec ce qu'un compositeur peut savoir (ou parfois deviner) des intentions de ses confrères. L'enquêteur à la recherche des 
phénotypes ne peut pas être un spécialiste de ces trois démarches dominantes; sa particularité est plutôt d'être un généraliste. Le besoin me paraît s'en faire sentir en musicologie comme en médecine.

25 - les génotypes. Toute enquête réfléchie révèle des analogies dans les pratiques d'engendrement de la musique, dans les sortes de manipulations des données sonores, ou dans les circonstances auxquelles ces pratiques sont liées. Ces processus peuvent engendrer des structures de surface extrêmement diverses, ils n'en constituent pas moins des lois d'organisation communes. Ces lois peuvent être explicites, codifiées, ou à peine formulables consciemment. Elles ne sont accessibles qu'à un niveau d'analyse qui soit déjà capable d'intégrer, au moins en une synthèse provisoire, les données collectées au niveau des phénotypes. C'est là que l'idée de musique expérimentale pourrait retrouver son vrai sens, c'est-à-dire non pas celui d'une aventure, mais de tests précis appliqués à des hypothèses. L'application de certaines lois de transformation à des données sonores peut ou non produire les phénotypes supposés au départ de l'expérience. Les réussites de certains pastiches programmés sur ordinateur, tout comme les progrès de l'interprétation de musiques anciennes par l'intégration de pratiques survivantes, donnent quelque espoir de résultats à cette direction de recherche d'une musicologie expérimentale.

26 - les archétypes sont le troisième niveau auquel il serait important d'avoir accès. Seule la concordance de multiples indices peut aider à les déterminer. Si les données acquises lors de l'enquête sur les deux niveaux précédents, les formes des phénotypes et les processus des génotypes, coïncident, leur accord peut signifier la présence d'une donnée première universellement liée à l'activité mentale de l'homme aux prises avec les sons. En d'autres termes, lorsqu'en des lieux et des temps différents et distants, un même phénotype apparaît, et que les circonstances de sa production relèvent partout d'un même génotype, il y a lieu de supposer qu'il est issu d'un schème naturel et mérite éventuellement l'appellation d'archétype. Si en revanche aucune convergence significative ne peut être mise en évidence, l'hypothèse d'archétypes sonores communs devra être reconsidérée.

27 La distribution entre phénotypes et génotypes introduit un découpage sémantique plus précis que celui d'Assafyev avec ses intonations, tout en explorant une voie assez comparable. En effet l'intonation a le même statut de figure fondatrice, mais d'une part le musicologue soviétique y voyait un cliché au succès historique variable, où on ne distingue pas assez entre figure sonore et processus productif, et d'autre part il tendait à privilégier l'émission vocale comme modèle de la musique, alors que la posture et le geste ont, à la source des musiques, un rôle au moins aussi fondamental, comme le montrent bien les travaux de G. Durand.

Outre l'hypothèse générale de l'existence au sein des pratiques culturelles - en l'occurrence musicales - d'une certaine quantité de données universelles, et l'interprétation proposée de cette universalité non pas comme le seul résultat d'influences réciproques mais comme le produit transformé de schèmes mentaux spontanés, naturels, plusieurs positions marquent les options initiales de cette recherche :

29 - compatibilité de cette tentative de retour à une musicologie comparée avec les études anthropologiques, et en particulier ethnomusicologiques. L'admission de certains « lieux communs » naturels ne travestit pas pour autant la musique en une activité réflexe, et la diversité, voire l'arbitraire, des pratiques culturelles, n'en sont pas moins 
pris en compte. La précieuse documentation fournie par les enquêtes ethnomusicologiques doit être complètement assimilée au sein d'une réflexion générale, et en aucun cas écartée ou minimisée.

30 - Bien que les outils conceptuels utilisés soient liés à la civilisation occidentale, et que l'intérêt pour l'universel soit lui-même dans la pure tradition de cette culture particulière, il n'est pas question de disqualifier ce point de vue à cause de son inévitable relativité, et avec lui toute entreprise scientifique. Le lien infrangible entre l'observateur et la chose observée ne saurait révoquer en doute toute possibilité d'observation. Les champs sémantiques locaux que nous révèlent les enquêtes peuvent ne pas coïncider entre eux sans que toute possibilité d'universaux soit récusée pour cela.

31 - Le dialogue sans cesse accru entre des musiques jusqu'alors éloignées géographiquement et/ou historiquement complique l'analyse des universaux en multipliant les emprunts croisés entre cultures, qui au demeurant ont toujours existé. J'en postule moi-même la légitimité avec des œuvres comme Phénix, Aliunde ou Guntur madu. Mais la possibilité même de tels emprunts plaide pour l'hypothèse d'archétypes où toute l'humanité puisse se retrouver. Le moment que nous vivons tend à accélérer fortement l'uniformisation des pratiques et des formes musicales sous l'effet d'une standardisation industrielle, qui exploite cyniquement la partie morte de ces lieux communs. Mais parallèlement le mouvement d'accumulation des connaissances de cultures musicales anciennes ou lointaines amorce au contraire désormais une décélération : les grandes redécouvertes de musiques anciennes sont moins fréquentes, et les grandes enquêtes de terrain ou bien ont déjà eu lieu ou bien sont devenues moins prometteuses du fait de la mondialisation de la culture. Le moment est donc venu de tenter de repérer ce qui, au sein des particularismes survivants et de la riche documentation sur les particularismes disparus témoigne musicalement d'une humanité commune. Le dépistage des archétypes par l'analyse devra procéder quelque peu à l'image des dépistages démographiques à l'aide de marqueurs génétiques subsistants.

32 - L'intervention de la zoomusicologie ne peut en aucune manière faire fi de l'évidente frontière entre animalité et humanité. Elle aura surtout pour rôle de fournir des indices confortant ou infirmant l'identification des archétypes musicaux qui se dégageront des enquêtes portant sur des corpus de musiques humaines. La convergence non seulement de phénotypes mais même de génotypes sonores issus du monde animal avec ces dernières a déjà été suffisamment constatée pour autoriser l'espoir de certains résultats (chapitre Zoomusicologie de Musique, Mythe, Nature, article Syntagms and paradigms in Zoomusicology etc.)

33 La plus grande universalité historique et géographique est nécessairement le cadre de la recherche. À l'ère de la fragmentation du savoir, aucun homme ne saurait embrasser la totalité des documents. Par ailleurs la solution que constituerait l'organisation d'équipes pluridisciplinaires n'est pas assurée dans une recherche de ce type. Aussi aije envisagé de procéder dans un premier temps, plutôt que par l'établissement d'une taxonomie de type linnéen, à l'aide de procédures hypothético-déductives. Elles seront appliquées à deux grands domaines de questionnements :

34 1) les catégories formelles «hors-temps » ou "en-temps », selon la terminologie de Xenakis, telles que : 
35

- les échelles musicales. Y a-t-il des traits universels dans leur constitution? Les modulos du tétracorde et de l'octave, leurs sources acoustiques et physiologiques.

- les modèles narratifs tels que la crise, l'énumération, le conflit, la variation évolutive etc.

- les modèles physiques tels que le reflux, l'écho, le ruissellement, l'éclatement, l'appel etc.

- l'opposition fond/figure (soli-tutti dans les formes responsoriales, mélodie accompagnée, plans orchestraux, proche et lointain etc.)

- la répétition (ostinati, refrains, reprises, formes Lied, Barform etc.) comme génotype universel.

2) les données de l'imaginaire musical. Quelle universalité trouve-t-on dans les caractères et la fréquence des associations :

- symboliques sexuelles (masculin-féminin dans les rythmes, les contours, les accents; tabous organologiques etc.)

- symboliques cosmologiques (heures, saisons, points cardinaux, astrologie etc.). Les associations propres à chaque culture répondent-elles à des constantes?

- émotionnelles (ethos, couleurs, circonstances etc.)

- sociales (castes, cérémonies, hiérarchies des genres, distinction sacré-profane etc.)

- entre l'acoustique du biotope et les musiques qu'on y pratique.

Bien évidemment, cette énumération de vastes domaines de recherche n'implique pas une impossible étude exhaustive de chacun, mais un tour d'horizon des gisements d'informations exploitables pour une synthèse comparative.

Les trois niveaux d'exploration correspondent à trois méthodes d'investigation dont on cherchera à rapprocher les résultats. La recherche des phénotypes fera appel aux procédures structuralistes telles que l'analyse distributionnelle, l'analyse paradigmatique, la sémanalyse, ainsi qu'à des catégories hypothétiques comparables aux intonations d'Assafyev ou aux prolongations dont Narmour a proposé la théorie.

8 La recherche des génotypes s'appuiera d'une part sur l'expérience de la composition musicale et d'autre part sur des hypothèses de psychologie cognitive (travaux de $\mathrm{S}$. McAdams, I. Deliège), en écartant celles dont la pertinence apparaîtrait trop liée aux seules musiques tonales.

9 La recherche des archétypes procédera d'une confrontation entre les résultats des deux autres niveaux, et utilisera aussi une herméneutique structurale issue entre autres de Cassirer, Eliade, Diel, Durand.

Pour aborder cette recherche par un domaine relativement facile, un premier projet pourrait concerner l'étude d'un des universaux les moins contestables : la répétition. Les phénotypes sont aisément repérables tant dans les signaux animaux que dans les musiques tonales et non-tonales, voire dans les vocalisations infantiles. Il s'agit par exemple de la reprise, de la duplication, du refrain (et avec lui de la forme scherzo et des formes strophiques), de la forme Lied, de la Barform, et de l'ostinato. Il sera intéressant de mesurer les rôles de différents processus "génotypiques" produisant ces phénotypes: par exemple celui de l'euphorie physique, celui de la mnémonique dans les traditions orales, celui d'une conception cyclique du temps etc. Le lien souvent observé entre l'acmé du système tonal et l'omniprésence de la répétition pourra être 
soumis à un examen critique à la lumière de la très abondante documentation nontonale. La pratique exceptionnelle de musiques totalement non-répétitives, en particulier dans les années 1950, pourra elle-même s'éclairer d'un jour différent une fois resituée dans un contexte de répétition aussi universelle.

Je crois qu'il est important de ne pas se résigner à voir la connaissance musicale se fragmenter à l'infini. Autant les avancées des spécialistes sont utiles, autant il convient sans cesse de les remettre en perspective pour que leur utilité soit perceptible. Si le risque de savoir beaucoup sur peu de chose, ou même tout sur presque rien, a jusqu'à maintenant épargné la musicologie, contrairement à d'autres domaines d'érudition, c'est sans doute une compensation à son manque d'autonomie: faute d'une doctrine propre, elle a davantage multiplié les paris épistémologiques généraux que les applications particulières de telle ou telle approche. Et faute de troupes très nombreuses, du moins en France, elle est loin d'avoir exploré tous les territoires qui relèvent de sa mouvance.

Mais cependant nous assistons à une hybridation générale qui se produit dans le monde entier à une vitesse explosive. La musicologie ne peut, me semble-t-il, se contenter d'explorer le passé : sa fonction de gardienne de Musée est quelque peu chahutée par le cataclysme des industries musicales, qui détruisent irrésistiblement les cultures particulières, devenues incapables et peu soucieuses de leur résister. Il me semble qu'au côté des spécialistes historiens, psychologues, sociologues etc. qui ont plus que jamais à faire avec l'afflux des cultures moribondes au chevet desquels ils restent penchés avec sollicitude, une petite place devrait être octroyée à des généralistes essayant de coordonner leurs points de vue, pour qu'une nouvelle musicologie comparée nous redonne l'envie de mieux comprendre ce qu'il y a de commun entre les hommes lorsqu'ils jouent avec les sons; et pour que la diversité de leurs jeux retrouvée au-delà des standards banals leur redonne un goût de vivre plus intense.

\section{NOTES}

1. MÂCHE, François-Bernard, Musique, mythe, nature ou les dauphins d'Arion, Paris, Klincksieck, 1983. [Réédition Adeam Musicae, 2015].

2. MÂCHE, François-Bernard, Musique au singulier, Paris, Odile Jacob, 2001.

3. MÂCHE, François-Bernard et SERROU, Bruno, François-Bernard Mâche. La musique, la nature, les oiseaux. Entretien avec Bruno Serrou, Paris, INA/Michel de Maule, 2006.

4. MÂCHE, François-Bernard, Cent opus et leurs échos, Paris, L'Harmattan, 2012.

5. MÂCHE, François-Bernard, « Méthodes linguistiques en musicologie », Musique en jeu 5 (1971), p. 75-91.

6. MÂCHE, Musique, mythe, nature ou les dauphins d'Arion, op. cit. 


\section{AUTEUR}

\section{FRANÇOIS-BERNARD MÂCHE}

François-Bernard Mâche, né à Clermont-Ferrand en 1935 de parents musiciens, a mené une double carrière universitaire et musicale : École Normale Supérieure (1955), Agrégation de Lettres Classiques (1958), Doctorat d'État ès Lettres (1980) ; et d'autre part, élève d'olivier Messiaen au Conservatoire National Supérieur de Musique et membre fondateur du Groupe de Recherches Musicales de Pierre Schaeffer (1958). Il a élaboré une théorie et une méthode personnelles de composition, centrées autour des idées de modèle et d'archétype. Pionnier de l'application des données linguistiques à l'analyse et à la création musicales, il a écrit plusieurs ouvrages tels que Musique, mythe, nature (Klincksieck, 1983 et 1991; Aedam Musicae 2015 ; ouvrage également traduit en anglais et en italien), Un demi-siècle de musique (L'Harmattan, 2000), Musique au singulier (Odile Jacob, 2001). Ses œuvres, au nombre d'une centaine, allient fréquemment les haut-parleurs et les instruments acoustiques. Il a été invité à les présenter et à enseigner dans plus de vingt pays de tous les continents. Il a reçu en 1977 le prix Italia, en 1988 le grand prix national de la musique et en 2002 le grand prix de la musique symphonique de la Sacem. Après avoir dirigé pendant plusieurs années le département de musique à l'Université de Strasbourg, il a enseigné comme Directeur d'études à l'École des Hautes Études en Sciences Sociales. Commandeur des Arts et Lettres et chevalier des Palmes académiques, il a été élu membre de l'Institut en 2002, au fauteuil précédemment occupé par Iannis Xenakis. 